\title{
Aneurisma de artéria poplítea com rotura e formação de pseudo-aneurisma
}

\author{
Popliteal artery aneurysm with rupture and pseudoaneurysm formation \\ H eraldo Antônio Barbato, M arcelo Tavares C unha, Alexandre Volpini C lauzo, \\ Ana Cláudia G omes Petisco, Fabio H enrique Rossi, Fernando D agli M alheiros, \\ Lannes Alberto Vasconcelos O liveira, N ilo Mitsuru Izukawa*
}

\begin{abstract}
Resumo
Paciente relatava história aguda de dor e edema em membro inferior direito há 5 dias, e a semiologia caracterizava pulsos poplíteos amplos e perfusão distal satisfatória. Exames laboratoriais mostraram insuficiência renal, e o exame de ultra-som duplex evidenciou um aneurisma de artéria poplítea à direita roto, com formação de pseudoaneurisma e um aneurisma de artéria poplítea à esquerda. 0 paciente foi submetido à exploração cirúrgica por via de acesso posterior, sendo evidenciado pseudo-aneurisma secundário a aneurisma de artéria poplítea roto e realizada endoaneurismorrafia com interposição de veia. A evolução pós-operatória foi boa. A ruptura de um aneurisma de artéria poplítea é uma complicação rara, e apenas um relato de formação espontânea de pseudo-aneurisma foi encontrado na literatura.

Palavras-chaves: Aneurisma, ruptura de aneurisma, pseudoaneurisma, artéria poplítea.
\end{abstract}

\section{Introdução}

0 aneurisma de artéria poplítea (AAP) tem como complicações mais freqüentes fenômenos embólicos, trombóticos e compressivos com riscos de perda de membro, trombose venosa profunda edistúrbios sensitivos e motores ${ }^{1,2}$. A ruptura do AAP é uma complicação incomum, porém constitui uma situação grave, com elevado índice de amputação ${ }^{3,4}$. A formação de um pseudo-aneurisma de artéria poplítea de maneira espontânea é um evento raro. N este artigo, relata-se 0

* Instituto Dante Pazzanese de Cardiologia, São Paulo, SP.

Artigo submetido em 03.01.06, aceito em 24.05.06.

\begin{abstract}
The patient presented with a 5-day history of acute pain and swelling in the right lower limb. On physical examination, wide popliteal pulses and satisfactory distal perfusion were observed. The laboratory work-up showed renal failure and the duplex ultrasound examination was suggestive of a ruptured right popliteal artery aneurysm with pseudoaneurysm formation and a left popliteal artery aneurysm. The patient underwent urgent surgery through a posterior popliteal approach. A ruptured aneurysm with pseudoaneurysm formation was found and repaired by endoaneurysmorrhaphy with interposition of a short saphenous vein segment. The postoperative period was unremarkable. Rupture of a popliteal artery aneurysm is a rare complication: a single report of spontaneous popliteal pseudoaneurysm was found in the literature.
\end{abstract}

Key words: Aneurysm, aneurysm rupture, pseudoaneurysm, popliteal artery.

caso de um paciente com AAP bilateral com formação de pseudo-aneurisma à direita, sendo submetido à correção com sucesso.

\section{Relato do caso}

H omem de 75 anos foi admitido com quadro de dor e edema súbitos no membro inferior direito com 5 dias de evolução. N egava história de trauma local. Ao exame, apresentava-sehemodinamicamenteestável, com edema doloroso à palpação, principalmente na região da panturrilha. Os pulsos poplíteos eram amplos, 0 pedioso direito, ausente, e os demais, normais. $\mathrm{N}$ ão apresentava alteração da perfusão. N os exames laboratoriais, apresentava creatinina sérica de $3,2 \mathrm{mg} / \mathrm{dl}$, uréia de81 mg/dl, hemoglobina de 11,2 g/dl, hematócrito de $32,3 \%$. O ultra-som Doppler revelou um pseudo- 
aneurisma de artéria poplítea direita de 3,8 x 3,2 cm com grande coágulo adjacente (Figura $1 \mathrm{~A}$ ) eaneurisma de poplítea esquerda de $3,4 \times 3,3 \mathrm{~cm}$. Em virtude da disfunção renal, optou-se pela não-realização de angiografia e indicou-se o tratamento cirúrgico. A via de acesso cirúrgico optada foi a posterior, sendo identificado um aneurisma roto com formação de pseudoaneurisma (Figura 1B e 2) e uma grande quantidade de coágulosadjacentes. A pós dissecção econtroledoscolos proximal edistal, foi realizada uma endoaneurismorrafia com interposição de segmento de veia safena parva (Figura 3). 0 paciente evoluiu no pós-operatório sem intercorrências. Após 3 meses, foi submetido à correção eletiva do AAP esquerdo.

\section{D iscussão}

0 AAP éo mais comum entre os periféricos ${ }^{1,5-7}$. A ocorrência de AAP contralateral foi relatada de 30 a $50 \%{ }^{2,8}$, podendo chegar até $70 \%{ }^{9}$. A ocorrência de aneurismas em outras artérias é freqüente, já podendo estar presentena épocado diagnóstico do AAP ou surgir ao longo dos anos durante 0 acompanhamento destes pacientes ${ }^{9-11}$. A origem dos AAP, tradicionalmente considerada como aterosclerótica, vem sendo considerada multifatorial ${ }^{9}$.

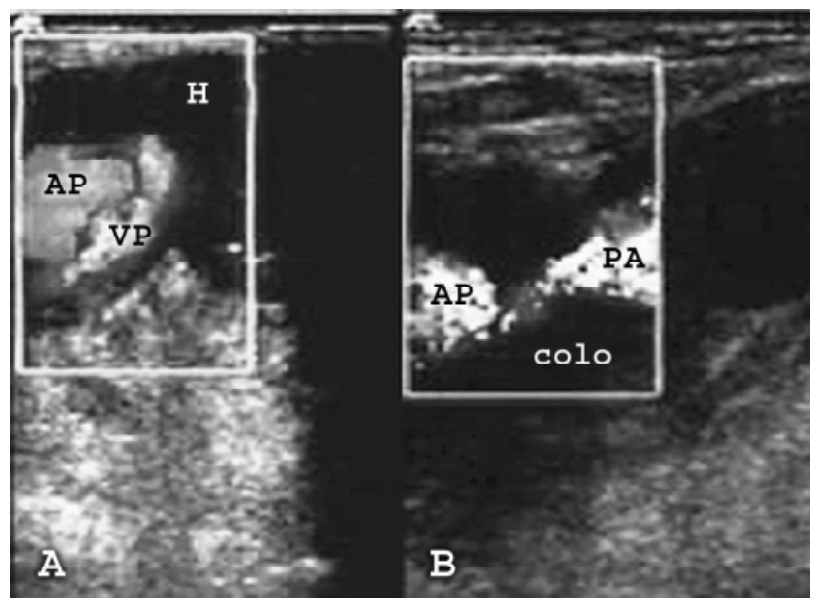

Figura 1 - D uplex scan daartéria poplítea direita. A) Aneurisma da artéria poplítea e trombo secundário à sua ruptura. B) Local da ruptura do aneurisma evidenciando fluxo no colo e no interior do pseudo-aneurisma

$\mathrm{AP}=$ artéria poplítea; VP = veia poplítea; $\mathrm{H}=$ trombo; $\mathrm{PA}$ = pseudo-aneurisma
Entre as complicações dos AAP, encontram-se a isquemia do membro, a ruptura e a compressão de estruturas adjacentes, como veias e nervos. A ruptura é uma complicação incomum, ocorrendo em 2,5 a 5,3\% dos casos $^{2,12-15}$. O s pseudo-aneurismas poplíteos são uma complicação tardia e rara, ocorrendo, geralmente, após trauma ou procedimentos ortopédicos. Apenas um caso de pseudo-aneurisma originário de ruptura espontânea de AAP foi encontrado na literatura ${ }^{16}$.

0 diagnóstico do AAP pode ser feito de diversas maneiras. Clinicamente, épossível identificar umamassa pulsátil na fossa poplítea. A suspeita clínica éconfirma-

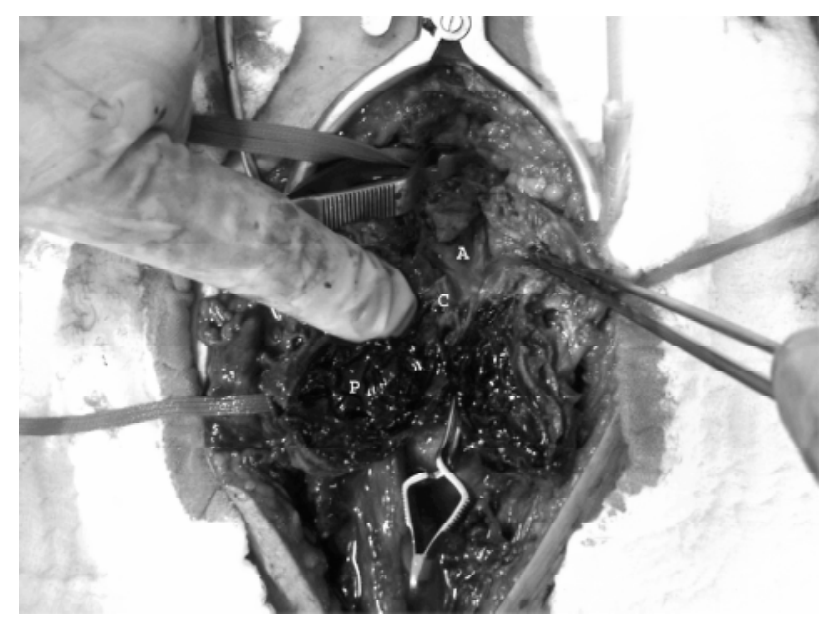

Figura 2 - Secção do pseudo-aneurisma

$\mathrm{A}=$ artéria poplítea; $\mathrm{C}=$ colo do pseudo-aneurisma; $\mathrm{P}=$ pseudo-aneurisma.

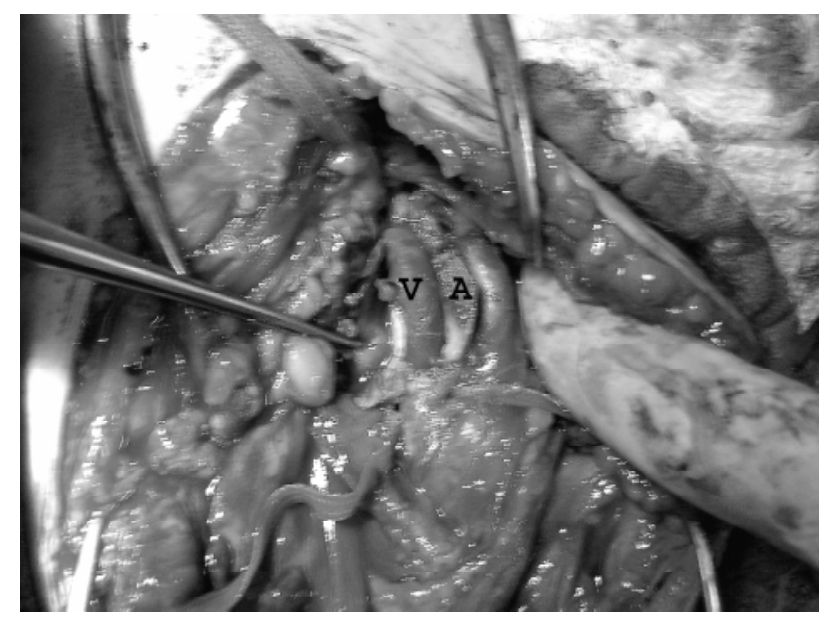

Figura 3 - Endoaneurismorrafia

$\mathrm{V}=$ enxerto de veia; $\mathrm{A}$ = "capa" do aneurisma. 
da com um duplex scan, que é capaz de definir o tamanho do aneurisma, a presença de trombos no seu interior e o fluxo sangüíneo, tanto no aneurisma quanto nos vasos distais. A tomografia computadorizada e a ressonância magnética são capazes defornecer informações semelhantes, porém são exames de maior custo e não disponíveis em todos os serviços.

0 tratamento do AAP é cirúrgico, através de um enxerto, preferencialmente venoso. A via medial pode ser optada realizando-se a exclusão do aneurisma, ou através de uma via posterior proceder a uma en doaneurismorrafia. 0 tratamento por via endovascular, através da colocação de endoprótese, foi relatado em um paciente com AAP roto e com alto risco cirúrgico, tendo sido alcançado um resultado satisfatório ${ }^{10}$. N o único caso semelhante ao nosso encontrado na literatura, optou-se por correção através de acesso medial com interposição de segmento de politetrafluoretileno (PTFE), condutajustificada pelalocalização supragenicular do AAP16. 0 tratamento endovascular de um AAP roto através da colocação de um stent revestido com PTFE (Hemobahn, W.L. Gore \& Associates, Flagstaff, EUA) também foi descrito com sucesso ${ }^{14}$, porém, com esse método, não é possível a retirada do material (coágulos, parede do aneurisma), que causa sintomas compressivos na fossa poplítea, além das limitações, até o momento, no uso destes dispositivos em lesões que ultrapassam a articulação do joelho.

\section{Referências}

1. Szilagyi DE, Schwartz RL, Reedy DJ. Popliteal aneurysms. Their natural history and management. Arch Surg. 1981;116:724-8.

2. Ramin EB, Christopher KZ. Popliteal and femoral artery aneurysms. In: C ameron JL, editor. C urrent surgical therapy. 6th. ed. St. Louis: M osby; 1998. p. 737-40.

3. $\mathrm{MCH}$ ugh JV. Spontaneous rupture of bilateral popliteal aneurysms. Ann Surg. 1951;133:131-4.

4. Barroy JP, Barthel J, Locufier J L, BosschaertsT, Goldstein M . Atherosclerotic popliteal aneurysms. Report of one ruptured popliteal aneurysm. Survey and analysis of the literature. J Cardiovasc Surg (Torino). 1986;27:42-5.
5. Duffy ST, Colgan MP, Sultan S, M oore DJ, Shanik GD. Popliteal aneurysms: a 10-yearsexperience. EurJ V ascEndovasc Surg. 1998;16:218-22.

6. Gaylis H. Popliteal aneurysms. A review and analysis of 55 cases. S Afr M ed J. 1974;48:75-81.

7. Vermilion BD, Kimmis SA, Pace W G, Evans W E. A review of one hundred forty-seven popliteal aneurysms with longterm follow-up. Surgery. 1981;90:1009-14.

8. D awson I, Sie RB, van Bockel JH. Atherosclerotic popliteal aneurysms. Br J Surg. 1997;84:293-9.

9. G raham LM . Femoral and popliteal aneurysms. In: Rutherford RB, editor. Vascular surgery. 5th ed. Philadelphia: WB Saunders; 2000. p. 1345-56.

10. Carpenter JP, Barker CF, Roberts B, Berkowitz H D, Lusk EJ, Perloff LJ. Popliteal artery aneurysms: current management and outcome. J V asc Surg. 1994;19:65-72; discussion 72-3.

11. D awson I, van Bockel JH, Brand R, T erpstra JL. Popliteal artery aneurysms. L ong-term follow-up of aneurysmal disease and results of surgical treatment. J Vasc Surg. 1991;13: 398-407.

12. I hlberg LH, R oth WD, Alback N A, Kantonen IK, Lepantalo $M$. Successful percutaneous endovascular treatment of a ruptured popliteal artery aneurysm. J V asc Surg. 2000;31: 794-7.

13. D avidovic LB, Lotina SI, Kostic D M , et al. Popliteal artery aneurysms. W orld J Surg. 1998;22:812-7.

14. Sie RB, D awson I, van Baalen JM, Schultze Kool LJ, van Bockel JH. Ruptured popliteal artery aneurysm. An insidious complication. Eur J V asc Endovasc Surg. 1997;13:432-8.

15. O liveira RSM, Ferreira DA, T erra JA Jr., Lenza RM, Filho ACA, N unes W . R upture of a popliteal artery aneurysm: case report and review of the literaturefor the past 50 years. J $V$ asc Bras. 2005;4:105-10.

16. Canbaz S, Ege T, Sunar H, Saygin G, Duran E. Bilateral popliteal artery aneurysms with ruptureand pseudoaneurysm formation on the left. Yonsei M ed J. 2003;44:159-62.

Correspondência:

$M$ arcelo $T$ avares $C$ unha

Avenida O nze de Junho, 582/31 - Vila Clementino

CEP 04041-002 - São Paulo, SP

Tel.: (11) 5579.2998, (11) 5085.4140, (11) 8281.9899

Fax: (11) 276.3199

E-mail: marcelotcunha@yahoo.com.br 